PTH-007

\section{WHOLE GUT REMOTE ENDOSCOPY': A NOVEL PROTOCOL FOR LUMINAL IMAGING USING COMBINED SMALL BOWEL AND COLON CAPSULE ENDOSCOPY}

doi:10.1136/gut.2011.239301.408

D Majumdar, ${ }^{1, *}$ R Sidhu, ${ }^{1}$ A J Lobo, ${ }^{1}$ M E McAlindon ${ }^{1}{ }^{1}$ Gastroenterology, Sheffield Teaching Hospitals NHS Foundation Trust, Sheffield, UK

Introduction Conventional endoscopy is uncomfortable, undignified, may require sedation and incur complications. Yet most procedures provide reassurance rather than identify relevant abnormalities. A sensitive diagnostic tool which is simpler, safer, less invasive and better tolerated, is needed. Capsule endoscopy (CE) was first developed to examine the small bowel, but provides images of oesophagus and stomach, and dedicated oesophageal and colon capsule endoscopes are in use. The authors describe a novel protocol using combined CE procedures to investigate the whole gastrointestinal tract.

Methods Patients had a 5 day low fibre diet and clear fluids only $12 \mathrm{~h}$ prior to admission. On the day of the test, they drank 21 Kleanprep between 19:00 and 21:00. At 22:00 they swallowed a small bowel capsule (SCE). At 08:00 the following day the data recorder was detached. A new data recorder was initiated and the patient swallowed a colon capsule (CCE) followed by $2 \mathrm{~L}$ Kleanprep. Two further doses of Fleet phospho-soda were administered and a bisacodyl suppository if the capsule was not excreted. One patient also had an oesophageal CE prior to discharge. Bowel cleanliness was described using a validated scale (1-4: excellent to poor). Results were described as median and IOR.

Results 27 patients (18.5\% male, median age 48 (IOR 31-57) underwent combined SCE and CCE to investigate altered bowel habit $(\mathrm{n}=20)$, abdominal pain $(\mathrm{n}=15)$, anaemia $(\mathrm{n}=5)$ and weight loss $(n=5)$. SCE visualised the stomach for 44.5 (IOR 15.5-87.3) and small bowel for 235 (IOR 137-370.5) min with a caecal intubation rate of a $88.9 \%$. CCE visualised the small bowel for 72 (IOR 45.5-147) and colon for 103 (IQR 60-216) min. Median colon cleanliness score was 3 (IOR 2-3) and complete examination rate was $66.7 \%$. Pathology was detected in $74.1 \%$ of patients: it was considered relevant to the indication in $40.7 \%$ of patients. Significant diagnoses included Crohn's disease $(n=8)$, polyps $(n=4)$ and NSAID colopathy $(n=1)$. Gastric erosions, polyps and altered blood in the stomach were noted in 3 patients. Of those with Crohn's disease, ileal disease was identified by colon CE in all cases.

Conclusion Single visit whole gut remote endoscopy with combination CE is feasible using the novel protocol described. The main indication was altered bowel habit with or without pain in patients $<50$ years of age in which the main imperative is to differentiate inflammatory bowel or coeliac disease from functional bowel disorders. CCE may suffice to provide evidence of
Crohn's disease in most cases without the need for SCE. Whole gut CE may become a first line GI diagnostic test, identifying patients for targeted conventional endoscopic biopsy and avoiding unnecessary invasive investigation in the future.

\section{Competing interests None.}

Keywords colon capsule, PillCam. 